\title{
Performance Estimation of HEVC/h.265 Decoder in a Co-Design Flow with SADF-FSM Graphs
}

\author{
Habib Smei ${ }^{1,2}$, Abderrazak Jemai ${ }^{1,3}$, Kamel Smiri ${ }^{1,4}$ \\ ${ }^{1}$ Laboratory LIP2, Faculty of Sciences of Tunis, University of Tunis El Manar, Tunis, Tunisie \\ ${ }^{2}$ General Directorate of Technological Studies, Higher Institute of Technological Studies of Rades, Ben Arous, Tunisia \\ ${ }^{3}$ University of Carthage, National Institute of Applied Science and Technology, Tunis, Tunisie \\ ${ }^{4}$ University of Manouba, Higher Institute of Multimedia Arts of Manouba, Manouba, Tunisie \\ Email:kamel.smiri@ismai.rnu.tn, habib.smei@isetsf.rnu.tn, abderrazek.jemai@insat.rnu.tn
}

How to cite this paper: Smei, H., Jemai, A. and Smiri, K. (2017) Performance Estimation of HEVC/h.265 Decoder in a Co-Design Flow with SADF-FSM Graphs. Int. J. Communications, Network and System Sciences, 10, 261-281.

https://doi.org/10.4236/ijcns.2017.1011016

Received: August 7, 2017

Accepted: November 11, 2017

Published: November 14, 2017

Copyright $\odot 2017$ by authors and Scientific Research Publishing Inc. This work is licensed under the Creative Commons Attribution International License (CC BY 4.0).

http://creativecommons.org/licenses/by/4.0/

c. (i) Open Access

\begin{abstract}
Multiprocessor System on Chip (MPSoC) technology presents an interesting solution to reduce the computational time of complex applications such as multimedia applications. Implementing the new High Efficiency Video Coding (HEVC/h.265) codec on the MPSoC architecture becomes an interesting research point that can reduce its algorithmic complexity and resolve the real time constraints. The implementation consists of a set of steps that compose the Co-design flow of an embedded system design process. One of the first anf key steps of a Co-design flow is the modeling phase which allows designers to make best architectural choices in order to meet user requirements and platform constraints. Multimedia applications such as HEVC decoder are complex applications that demand increasing degrees of agility and flexibility. These applications are usually modeling by dataflow techniques. Several extensions with several schedules techniques of dataflow model of computation have been proposed to support dynamic behavior changes while preserving static analyzability. In this paper, the HEVC/h.265 video decoder is modeled with SADF based FSM in order to solve problems of placing and scheduling this application on an embedded architecture. In the modeling step, a high-level performance analysis is performed to find an optimal balance between the decoding efficiency and the implementation cost, thereby reducing the complexity of the system. The case study in this case works with the HEVC/h.265 decoder that runs on the Xilinx Zedboard platform, which offers a real environment of experimentation.
\end{abstract}

\section{Keywords}

HEVC, h.265, Performance Estimation, SDF, SADF, SADF-FSM, Embedded 
Systems

\section{Introduction}

The evolution of digital video industry is being driven by continuous improvements in processing performance, availability of higher-capacity storage and transmission mechanisms. The increasing complexity of embedded applications deployed on Multiprocessor Systems-on-Chip (MPSoC) ranging from multimedia applications and telecommunications to aerospace applications has raised problems related to performance estimation and evaluation. The designer must have a view of the execution time of the various functions of the system to be designed, the memory size, the energy consumed, the silicon space of the hardware components and other necessary parameters to take decisions for architectural solutions. Performance evaluation in embedded systems can be carried out by different methods; either by measurement, by simulation or by analytical methods. Analytical methods are based on mathematical models to represent the application on one hand and the hardware platform on the other hand and then to apply performance estimation algorithms to analyze them.

Although these analytical approaches are less precise than approaches based on measurements and simulation, they are characterized by their speed and high level of abstraction allowing the designer to make quick decisions for the architectural choices of the system to be designed. Analytical methods represent the system to be conceived as a set of competing processes or actors linked through communication channels and called Model of computation. A Model of Computation (MoC) determines the rules that are used for computation inside processes and communication between processes. Models of computation allow abstracting the implementation of computation and communication in the system. Once system functionality is expressed using a MoC, this model can be subjected to transformations and analysis to reach an efficient implementation. There are many MoCs in the literature [1] [2] [3]. One of the most MoCs used to model streaming applications is Synchronous Data Flow (SDF) [4] [5]. Our work is concerned by the use of a variant of SDF graphs called SADF to estimate analytically the maximal achievable throughput of a multitask application under design. SDF Graphs (called also multi-rate regular dataflow graphs), initially proposed by Lee [6], are directed graphs where edges represent one-to-one data channels and whose vertices represent actors or tasks that operate on that data. As soon as all the input data have arrived, these actors begin their execution, after which they produce their output data. As long as such a data flow model is analyzable, performance guarantees can be obtained at design time.

\section{Related Works}

Synchronous Data Flow graphs are used in several works that deal with modeling, performance estimation and throughput analysis of MPSoC applications. S. 
Stuijk [7] is the first person who proposed an SDF graph to map a multimedia application on NoC-MPSoC platforms in order to minimize the resource usage. The flow begins with an application-aware SDF that is gradually transformed to handle resource sharing over a multi-tile architecture. In [8], authors use timed SDF graph to model NoC architectures with predefined guaranteed of bandwidth and a maximum latency. Several predictable arbitration mechanisms of MPSOC and NoC have been used, such as Round robin, TDMA, and Static sharing. In [9], authors studied the use of SDF in performance estimation after task migration from software to hardware. In [10], authors proposed an approach that uses Resource Manager (RM) actor to analyze applications that are modeled with SDF graphs. RM is a task responsible for resources access (critical or not). The designer reserves for the RM a whole execution node (CPU, memory, bus...) which increases the cost of the total MPSoC system. In [11], Wiggers et al. proposed a solution that exploits the SDF graphs to compare the throughput obtained with the target throughput of the application. SDF is used for the modeling sharing multi-port memory tiles in order to generate the best allocation. In [12], authors use SDFs to estimate the worst-case performance of a system before implementation. They developed a generic communication assistant module for multi-processors and multi-applications systems. In [13] [14], authors use FSM-SADF in order to model dynamic applications with multiple execution scenarios. Each scenario (behavior) is modeled by an SDF, and the FSM represents the possible orders in which active scenarios occur. Astochastic version of the SADF model is studied in [15]. In addition, in [16] homogeneous SDF graphs are considered (graphs in which all consumption and production rates are equal to one) to use SDF behavior. Only the execution times of affixed collection of actors can vary with scenarios. The approach presented in [17] is the most related to this work. It uses essentially the same model of computation, Scenario-Aware Dataflow Graphs (SADF). It introduces an analysis technique that works by building up a global state-space representation of the detailed behavior of the graph across sequences of scenarios. Transitions are at the level of individual firings of actors. This tends to lead to very large state spaces and tractability issues with larger models. [18] deals with scenarios of SDF behavior, but in their case only homogeneous SDF graphs are considered (graphs in which all consumption and production rates are equal to one), and only the execution times of affixed collection of actors can vary with scenarios. In [19], authors tried to find linear upper bounds on transient behavior of an SDF; this allows the behavior of an SADF to be analyzed.

\section{Background}

A Co-design flow of embedded systems [20] consists of a set of steps beginning with a requirements specification and ending with the integration of the software and hardware into silicon chip. Among all stages of a Co-design flow, performance estimation is performed to carry out measurements relating to execution time, energy consumption, memory space of the various functions and other 
necessary information for co-design steps.

Specification is usually represented as a software application coded with a high level language such as $\mathrm{C} / \mathrm{C}++$, Matlab, Sytemc. This specification is run on a host machine in order to test its functionalities and further understand the specificities of the whole system. Once this specification is tested, a profiling step [21] [22] is usually performed to define a profile of each entity of the system and establish a call graph function as well as other information such as execution time, size and type of data Exchanged. All this information is a parameter whose designers use to define architectural choices either in the modeling phase and partitioning phase of the Co-design flow. Once the modeling is done, a verification of the established model is required. Several methods, languages and tools are available to designers to do this verification. The choice of tools and approaches depends essentially on the nature of the application to be modeled (for example, data flow oriented or control flow oriented), but also depends on the experience of the design team and the availability of tools grasped.

Usually the verification step is performed by simulation, hardware emulation or by formal methods (e.g. Model Checking, Theorem Proving...). But when applications become increasingly complex (e.g. Multimedia applications) and architectures are increasingly powerful (e.g. MPSOCs), the use of these traditional verification methods on complex embedded systems appears increasingly inefficient. In fact, the use of traditional methods of verification with these new systems consumes either an intolerable processing time (e.g. logic simulation), or an exaggerated memory size (e.g. Model Checking). For this purpose, designers have recourse to new modeling and verification methods, namely Model of Computation (MOC).

\subsection{Data Flow Model of Computation}

A model of computation is a representation of the application as a function of the data dependencies that exist between its different functional blocks. The application will thus be represented as a network of several tasks whose application is composed of. This type of representation makes it possible to facilitate the knowledge of the intrinsic parallelism of the application and of the memory space that the application will need. Kahn process networks [23] were at the origin of the data flow paradigm. A Kahn process network is a network of actors connected by infinite (unbounded) channels of the FIFO type and able to store data tokens. A data token is an atomic data fragment (can not be split). Kahn also established a formal representation for the Kahn Process Network. An actor can therefore be described as a functional process that produces a sequence of data tokens from another data token sequence. The network of data flow processes inherits its formalism from the Kahn process networks, but associates with each actor a set of fusion rules that give the tokens necessary for an actor to trigger. This allows for a more in-depth analysis of the network using a set of properties. To allow further analysis, the Synchronous Data Flow (SDM) model takes the semantics of the data-flow process network and restricts its expressivity 
by specifying the data production/consumption rate as an integer value for each actors interconnection. Such information allows certifying the application to be deadlock free and to compute a statically schedule and computer memory requirements. In order to allow the expressiveness of the rather restrictive SDF model, several models were issued. The Boolean Data Flow Model (BDF) [24] aims at introducing switching and selection instructions into the SDF model. Boolean data flow (BDF), for instance, extends the SDF model of computation with two special actors called switch and select. The first one reads one input token and forward it to one of two possible outputs. The output to select is specified by a second Boolean input port. In the same way the select actor reads a token from one of two possible inputs and forwards it to a single output port. Already these simple extensions together with unbounded FIFOs are sufficient to generate a Turing complete model of computation. Integer-controlled data flow (IDF) [25] is an extension of BDF in that the switch and select actors can have more outputs or inputs, respectively. Whereas this helps to simplify the application models, it does not offer further expressiveness, as BDF is already Turing complete. Cyclo-dynamic data flow (CDDF) [26] aims to enhance the analysis capabilities of dynamic data flow descriptions. Its major idea is to provide more context information about the problem to analysis tools than this is done by $\mathrm{BDF}$. For this reason, an actor executes a sequence of phases whose length depends on a control token. The latter can be transported on any edge, but must be part of a finite set. Each phase can have its own consumption and production behaviour, which can even depend on control tokens. Several restrictions take care that the scheduler can derive more context information, as this would be the case for BDF graphs. For instance, a CDDF actor is not allowed to have a hidden internal state.

\subsection{Synchronous Data Flow}

Synchronous dataflow (SDF) [27] [28] network is composed of actors that are connected by FIFO channels. When an actor fires, it consumes tokens from input channels and produces tokens on output channels. Firings of an SDF actor create a process. The actor's firing rule specifies how many tokens are consumed on each input and how many will be produced on each output. In SDF, the number of tokens consumed on each input in every firing is constant, ie the firing rule remains the same.

The constant number of chips consumed and produced makes possible to make very efficient statics schedules.

Figure 1 presents an SDF graph with three actors. Consumption and production rates of tokens are labeled on each channel. For example, the consumption and production rates in channel ch3 are respectively TA13 and TA31.

To construct a static schedule for an SDF graph, we must follow two steps. The first step is to determine how many times each actor should fire during iteration. Iteration is a series of actor firings that return the channels to their original state. The number of tokens in a channel is the same before and after 


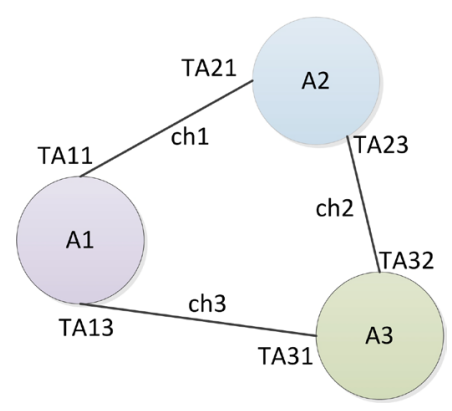

Figure 1. Example of a SDF graph.

iteration. To accomplish the first step, we must solve the set of balance equations. Balance equations state that production and consumption of tokens must be equal on all channels. The balance equations for the SDF graph the Figure 1 are shown below.

$$
\begin{aligned}
& F A 1 \times T A 11=F A 2 \times T A 21 \\
& F A 1 \times T A 13=F A 3 \times T A 31 \\
& F A 3 \times T A 32=F A 2 \times T A 23
\end{aligned}
$$

$F A 1, F A 3, F A 2$ are integers showing how many times actors $A 1, A 2$ and $A 3$ fire in a single iteration. They form a firing or repetition vector. The least positive integer solution is taken. For example if $T A 11=2, T A 13=2, T A 31=3$, $T A 32=3, T A 21=6$ and $T A 23=6$ then $F A 1=3, F A 3=2$ and $F A 2=1$. We must resolve this equation: $F A 1 \times 2=F A 2 \times 6$ and $F A 1 \times 2=F A 3 \times 3$ and $F A 3 \times 3=F A 2 \times 6$. We have as solution $F A 1=3, F A 3=2$ and $F A 2=1$

If we have as solution zero (the only solution), then the SDF graph is said to be inconsistent. This means that production and consumption of tokens cannot be balanced on all channels. As a result, the executions of an inconsistent SDF graph.

SDF graph is an analyzable model, while certain more expressive dataflow models such as Dynamic Dataflow or Kahn Process Networks [23] are known to be undecidable; they couldn't be used directly for such purpose. SDF graphs restrict their actors to produce and consume data with fixed rates per firing or, in case of CSDF (Cyclo Static Data Flow) with fixed periodic patterns. Many modern streaming applications, such as video or audio codec's use advanced compression and decompression algorithms and consequently have dynamic behavior. So, they can't be represented by a classic SDF graph. Extensions of the SDF graph are then necessary to cover such applications. Indeed, the dynamism originates from various modes of operation in which resource requirements are considerably different. As an example the HEVC codec, the case study on this work. This CODEC divides a video stream into frames which itself is divided into blocks called CTU and uses different coding schemes for each CTU, depending on its content [29] [30] [31]. SDF or CSDF cannot capture dynamic switching between frame types except by using an overly pessimistic common pattern for any frame type. Scenario-based or mode-based design [13] [14] is an approach in which the dynamic behavior of an application is considered as a set 
of separate behaviors, called scenarios or modes. Each scenario is static and predictable in performance and resource utilization. It can therefore be treated by traditional methods. However, some other difficulties need to be addressed such as predicting scenarios and handling transitions between them.

In this work, we use the Scenario-Aware Dataflow (SADF) [14] generalization of CSDF (Figure 2, Figure 3), which is based on scenarios to model the embedded software and the target hardware platform. SADF characterizes each scenario or individual mode by a specific SDF graph that models tasks with constant worst-case execution times. A finite state machine (FSM) is used for the Transitions between scenarios.

The HEVC Decoder is a dynamic application with several execution scenarios. Therefore, it cannot be modeled by SDF. The FSM-based-SADF extension is the suitable to model the application for a specific class of bitstream. Indeed, for a given class (a fixed frames resolution), it is possible to have four possible configurations (AI, RA, LP, LB) and several execution scenarios. The management of the various scenarios is carried out using a finite state machine (FSM).

\section{Our Approach of MPSoC Co-Design Oriented Performance Evaluation}

Our goal for using SDF in our Co-design flow is the performance estimation. It enables us to carry out measurements relating to execution time, energy consumption, memory space of the various functions and other necessary information for Co-design steps. Figure 4 presents our approach of MPSoC Co-design oriented performance evaluation. This approach is structured around four models: Functional Model (MF) of the application that describes the application behavior, Architecture Model AM which describes the target platform in number of processors, cache memory, communication system Bus/NoC..., Constraint Model (CM)in which constraints are specified and Performance Model (PM) that is used to provide performance metrics such as execution time and throughput. The two new models that we introduce in this approach are the PM and the CM models. These are two key models that guide the designer in the architectural choices during the Co-design process of the MPSoC system. The Constraint Model (CM) describes the functional constraints of the application and non-functional constraints of the target platform. The Performance Model

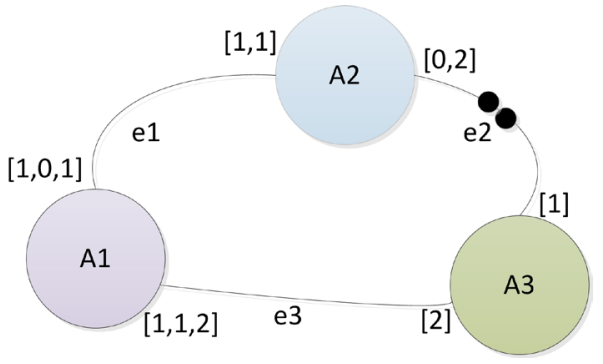

Figure 2. Example of a CSDF graph. 


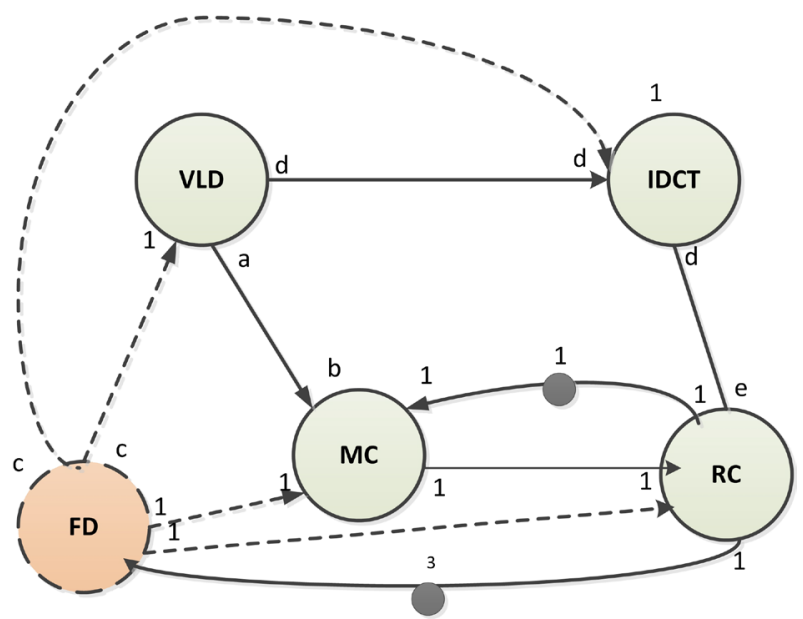

Figure 3. Example of a SADF graph.

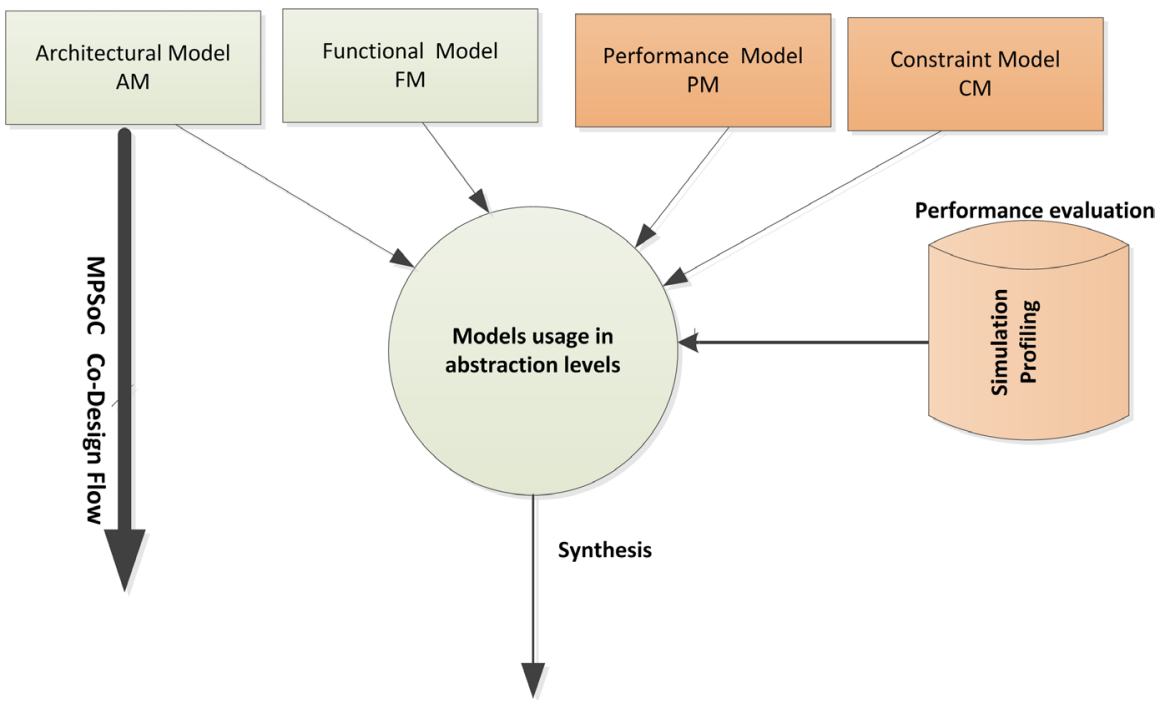

Figure 4. Approach of MPSoC Co-design oriented Performance estimation.

PM describes a performance evaluation of the system in every abstraction level. Every refinement of system is followed by a refinement of the correspondent PM model. The PM is enriched by results gotten by techniques and tools of performance evaluation. The PM is more precise to the lowest abstraction levels. It takes into account the task parallel model, Software/Hardware partition, Operating System Real Time (RTOS), hardware architecture (type and number of the processors, size of RAM and size of cache memory) and communication system (Bus, NoC).

Since at each step of the design of an MPSOC there is often a huge set of solutions that should be explored, then a good performance estimation technique is required to identify no efficient solutions at early steps. We propose an approach where, at each level of abstraction, a functional model of the application is mapped on a model of the target platform to estimate the performance. If performance values obtained do not meet the application constraints then other design solutions 
should be explored at this abstraction level otherwise additional details will be added to these models.

Starting from a pure functional model of the application, the following details are gradually added: the number of processing elements, the HW/SW partitioning, the hardware topologies, scheduling techniques used by the embedded OSs, Communication network architecture, HAL, etc. Both analytic and simulation techniques are used during the estimation.

\section{Case Study: HEVC/h.265 Decoder}

The HEVC standard [29] [30] is based on hybrid video coding based blocks. It implements the concept of block partitioning, which divides the image into blocks. Each block is predicted using either intra-frame or inter picture prediction. Intra-frame exploits spatial redundancy between the blocks within an image. Inter-picture uses the temporal redundancy between pictures. The prediction error is made by the difference between the original image and the predicted image in the case of both intra- and inter-picture prediction. The resulting prediction error is transmitted to the transform coding means followed by quantization and entropy coding. In the previous version of the standard HEVC (AVC, h.264) [31], the division of a frame is done according to $16 \times 16 \mathrm{ma}-$ cro-blocks. In HEVC, h.265, a frame can be divided into "coding tree blocks" (CTBs). Depending by an encoding setting, the size of the CTB can be of $64 \times$ $64,32 \times 32$ or $16 \times 16$. Indeed, several studies have shown that bigger CTBs provide higher efficiency (but also higher encoding time). Each CTB can be split recursively, in a quad-tree structure, in $32 \times 32,16 \times 16$ down to $8 \times 8$ sub-regions, called coding units (CUs).

In HEVC, CUs are the basic unit of prediction. Usually smaller CUs are used around detailed areas (edges and so on), while bigger CUs are used to predict flat areas. Each CU can be recursively divided into Transform Units (TUs) or Prediction Units (PUs) with the same quad-tree approach used in CTBs. Unlike AVC that used mainly a $4 \times 4$ transform and occasionally an $8 \times 8$ transform, HEVC has several transform sizes: $32 \times 32,16 \times 16,8 \times 8$ and $4 \times 4$. The transforms are based on DCT (Discrete Cosine Transform) except for intra $4 \times 4$, when transform is based on DST instead (Discrete Sine Transform) because several tests have evidenced a small improvement in compression. The HEVC decoder takes as an input a compressed file called bit-stream from which it extracts and decodes all the syntax elements, and then constructs each frame of the original video sequence. Figure 5 illustrates a common architecture of the HEVC decoder.

The decoding process can be divided into four main stages (Figure 5). The first stage is the entropy decoding that parses and decodes the bitstream file. It extracts relevant data such as reference frame indices; intra-prediction mode and coding mode. This information is transmitted to their respective modules. The second stage is called reconstruction step, which contains the inverse quantization (IQ), inverse transform (IT) and a prediction process. 


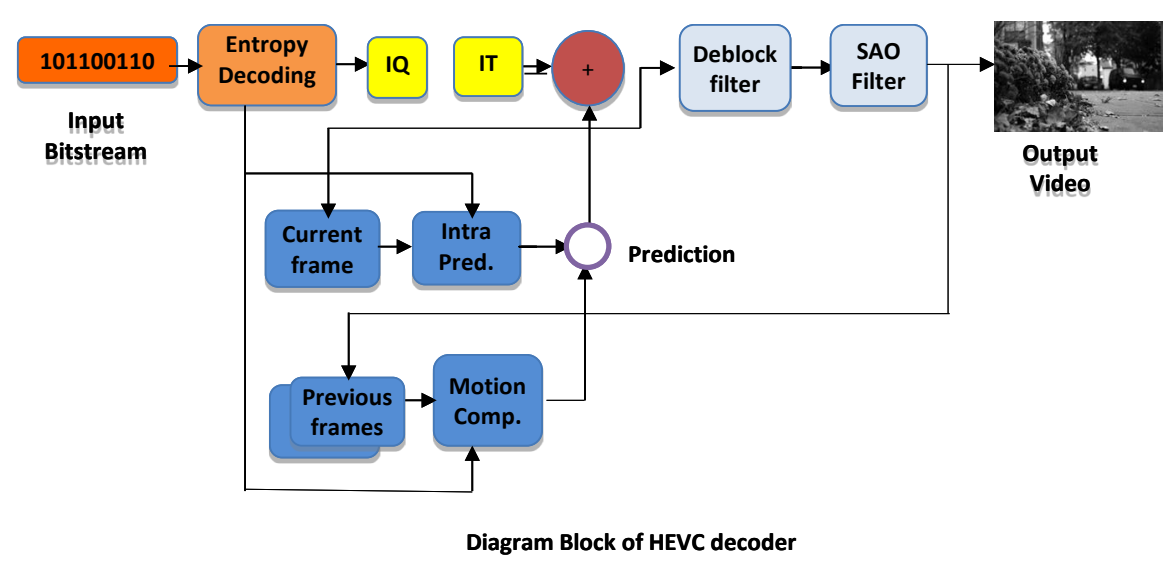

Figure 5. Block diagram of HEVC Decoder.

Quantized and transformed coefficients are handled, respectively, by the inverse quantization (IQ module in Figure 5) and the inverse transform (IT module, in Figure 5). The prediction process can be either intra-prediction or motion compensation (inter-prediction). The intra prediction block operates when the frame is an I frame. The inter prediction block operates when the frame is a $\mathrm{P}$ or B frame. The next step, before filtering is the storage of the reconstructed samples, the residue added to the predicted samples. This latter, will be used as references by the intra-frame or the inter-frame prediction modules.

The third step is the Loop filter composed by two filters blocks. The first is Deblocking Filter (DF) applied at the boundaries of the reconstructed blocks. It reduces the mean sample distortion of the decoded frame compared to the original one. The second is a new filter introducing with HEVC called Sample Adaptive Offset (SAO). It transmits offset values that can either resemble to the intensity band of pixel values (band offset) or the difference compared to neighboring pixels (edge offset).

\section{FSM-SADF Model of HEVC/265 Decoder}

To model an application with an SDF graph, the designer must have at his disposal information that concern the application, its functions (actors), the exchanged data (tokens) and their sizes, the size of the data used in each function and constraints to which the application is subject. This information can be extracted by performing a fine profiling step of the application. We have thus performed a profiling step that combines manual profiling with the use of available profiling tools such as Valgrind [32], Gpr of [33], mempr of [34]. Sequences used in this work are extracted from reference sequences and configurations proposed by the JCT-VC [35]. The sequences are divided into 6 groups (Classes). Group A corresponds to sequences with a resolution of $2560 \times 1600$ pixels, group B contains sequences with a resolution of $1920 \times 1080$, group C consists of sequences with a resolution of $832 \times 480$ pixels, sequences of groups $D$ have a resolution of $416 \times 240$ pixels and Group E consists of sequences with a 
resolution of $1280 \times 720$. The last group, $F$ consists of sequences with a resolution of $1024 \times 768$.

Our work is based on the HEVC HM Test Model [36]. This is an open source project under BSD license. It is intended for the implementation of an efficient HEVC C++ decoder. The version used in this work has been downloaded from [36] and it is compliant with the HEVC standard. Our profiling strategies are described in previous work [22] [30].

\subsection{Actors Identification and Dependencies}

To determine actors of the system and the relations between them, we check the behavior of the decoder described above by carrying out a parametric analysis of the application.

According to the profile stage, we identify eight actors as presented in Table 1. Each actor represents a set of $\mathrm{C}++$ classes in the $\mathrm{HM}$ test Model application.

\subsection{Number and Size of Data Exchanged (Tokens Exchanged)}

The number and size of the tokens exchanged between the actors is determined by following the WCET principle.

It is necessary to observe the data exchanged in the different configurations and to choose the largest number and the largest size. We must therefore define this information for the different classes $(\mathrm{A}, \mathrm{B}, \cdots, \mathrm{F})$. We will take as an example the class $\mathrm{C}$ that corresponds to the $832 \times 480$ resolution which corresponds to 399,360 pixels. So the size will be $399,360 \times 4=1,597,440$ bytes. According to the rule of the worst case, the size of CTU that will be chosen is $16 \times 16(256)$. So the maximum number of CTUs (tokens) exchanged will be: $(832 \times 480) / 256$ or 399360/256 = 1560 CTU (case of class C).

The data exchanged between the different actors of the HEVC CODEC are:

- Residual CTUs that are processed by IQ, IT actors. The maximum size of a CTU is $64 \times 64$ or 4096 pixels or 16,384 bytes.

- Intra-prediction data (from the ED block to the Intra-prediction), it can be at most a CTU that is: 16,384 bytes.

Table 1. Actors of HEVC Decoder.

\begin{tabular}{cc}
\hline Actor & Functionnal Block \\
\hline ED & Entropy Decoding \\
IQ & Inverse Quantification \\
IT & Inverse Transform \\
IP & Intra Prediction \\
MC & Motion Compensation \\
RC & Reconstruction \\
DF & Deblocking Filter \\
SAO & SAO Filter \\
\hline
\end{tabular}


- Inter-prediction data (from the ED block to the Inter-prediction block or MC block) composed of the motion vectors. Each motion vector is encoded with 12 bytes (array of 3 integers). Indeed, the motion vector is composed of 3 parameters, the origin, the angle and its length. Each one coded by 1 byte.

- An unfiltered image (from the RC block to the Loop Filter block), 399,360 pixels $^{\star} 4$ bytes or $1,597,440$ bytes (size of a frame for class C).

- A full filtered image (from the Loop Filter block to the prediction blocks, IP and MC), 399,360 bytes or 1,597,440 bytes (size of a frame for class C).

- Useful data for the Loop Filter block (from the ED block to the SAO filter). It can be maximum 5 bytes.

\subsection{Number of Motion Vectors}

The number of motion vectors is always less than or equal to the number of CTUs (it will be equal if all the CTUs that exist in the current image also exist in the reference image even if they change positions) because a vector of Movement carries information about a CTU that has a copy in the reference image. To do this, we can fix the Maximum number of motion vectors in a frame at 1560 (the number of CTUs in a class $C$ frame).

This will pose a modeling problem since it is difficult to create a scenario for each case (i.e. 1561 cases for class C, for example). According to a work in [37], the number of blocks that have motion vectors in a frame obeys a probabilistic law. We rely on the values proposed in the following table (Table 2) and the curve of Figure 6.

Actually, and observing this probability curve, we can note that this charts has a spike between values $30.3 \%$ and $60.6 \%$. So it is possible to limit the choice of the values representative of the possible cases of the motion vectors, which are very numerous as it is already mentioned. It can be limited to values in this interval and moreover one will try to distribute the chosen values along the chosen portion of the interval to be near to the real values whatever the case (the real value falls always close to one of the approximate values).

\subsection{Scenarios Determination}

An application that changes its mode of operation according to its input is a dynamic application. Therefore, it must be modeled with dynamic modeling. Each use case will have its own parameters (execution time, memory size, number of tokens exchanged....) and therefore corresponds to an execution scenario. The HEVC decoder is an example of dynamic applications with multiple execution scenarios. Indeed, for a given class (A, B, C, .., F), the operation mode of the decoder depends on three parameters:

- The type of frame to be decoded (I, P or B).

- The number of motion vectors in this frame ( $\mathrm{P}$ and $\mathrm{B})$.

- The number of blocks that do not have copies in an I frame. 


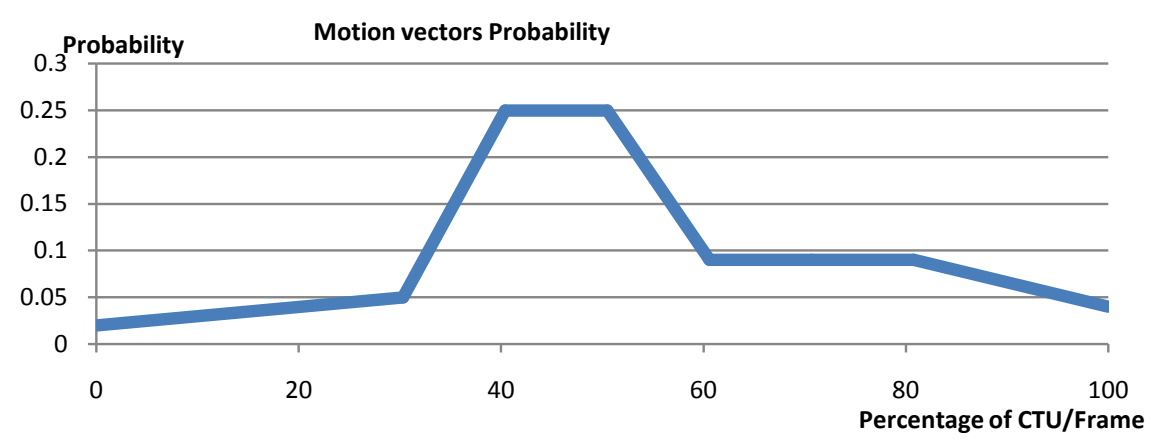

Figure 6. Probability of the numbers of motion vectors.

Table 2. Percentage and number of CTU/frame with motion vectors

\begin{tabular}{cccccccc}
\hline $\begin{array}{c}\text { \% of } \\
\text { CTU/frame }\end{array}$ & $\begin{array}{c}\text { Number of } \\
\text { CTU/Frame } \\
\text { (Class A) }\end{array}$ & $\begin{array}{c}\text { Number of } \\
\text { CTU/Frame } \\
\text { (Class B) }\end{array}$ & $\begin{array}{c}\text { Number of } \\
\text { CTU/Frame } \\
\text { (Class C) }\end{array}$ & $\begin{array}{c}\text { Number of } \\
\text { CTU/Frame } \\
\text { (Class D) }\end{array}$ & $\begin{array}{c}\text { Number of } \\
\text { CTU/Frame } \\
\text { (Class E) }\end{array}$ & $\begin{array}{c}\text { Number of } \\
\text { CTU/Frame Probability } \\
\text { (Class F) }\end{array}$ & \\
\hline 0 & 0 & 0 & 0 & 0 & 0 & 0 & 0.02 \\
30.3 & 4848 & 2454.30 & 472.68 & 118.17 & 1090.80 & 930.816 & 0.05 \\
40.4 & 6464 & 3272.40 & 630.24 & 157.56 & 1454.40 & 1241.088 & 0.25 \\
50.5 & 8080 & 4090.50 & 787.80 & 196.95 & 1818.00 & 1551.360 & 0.25 \\
60.6 & 9696 & 4908.60 & 945.36 & 236.34 & 2181.60 & 1861.632 & 0.09 \\
70.7 & 11312 & 5726.70 & 1102.92 & 275.73 & 2545.20 & 2171.904 & 0.09 \\
80.8 & 12928 & 6544.80 & 1260.48 & 315.12 & 2908.80 & 2482.176 & 0.09 \\
100 & 16000 & 8100 & 1560 & 390 & 3600 & 3072 & 0.04 \\
\hline
\end{tabular}

The number of scenarios will therefore depend on the combinations of frame types, number of blocks in a frame with motion vectors (for P and B frames), and blocks with copies for I frames

\section{Experimental Results}

\subsection{Experimental Platform}

Zedboard platform [38] (Table 3) is an evaluation platform based on a Zynq-7000 family [39]. It contains on the same chip two components. The first is a dual-core ARM Cortex MPCore based on a high-performance processing system (PS). It can be used under Linux operating system or in a standalone mode. The second is an advanced programmable logic (PL) from the Xilinx 7th family that can be used to hold hardware accelerators in multiple areas. The two parts (PS and PL) interact between them by using different interfaces and other signals through over 3000 connections. Available four 32/64-bit high-performance (HP) Advanced eXtensible Interfaces (AXI) and a 64-bit AXI Accelerator Coherency.

\subsection{Test Sequences}

As an effort to carry out a good evaluation of the standard, the JCT-VC developed a document with some reference sequences and the codec configuration, 
Table 3. Zed board technical specifications.

\begin{tabular}{cl}
\hline Component & \multicolumn{1}{c}{ Characteristics } \\
\hline $\begin{array}{c}\text { Processeur ZYNQ-7020 AP SOC } \\
\text { XC7Z020-7CLG484CES }\end{array}$ & 2 ARM Cortex A9 cores at 667 MHz \\
Memory & 512 MB DDR3, 256 MB Quad-SPI Flash and SD Card \\
Communication & $10 / 100 / 1000$ Ethernet, USB OTG and USB UART \\
Extension & FMC (Low Pin Count) and 5 Pmod headers $(2 \times 6)$ \\
Display & HDMI output, VGA output and 128 $\times 32$ OLED \\
Input/Output & 8 switches, 7 push buttons and 8leds \\
Current and Voltage & 3.0 A (Max) and 12V DC input \\
Certification & CE and RoHS certifier
\end{tabular}

which should be used with each one [35]. The sequences are divided into 6 groups (Classes) based on their temporal dynamics, frame rate, bit depth, resolution, and texture characteristics

A subset of six video sequences was selected from this list. These six video sequences were selected from classes A to F. Detailed descriptions of the sequences are given in Table 4.

\subsection{The SDF3 Tool}

The open-source SDF3 tool set [40] used in this work offers an SADF graph generation algorithm that constructs random SADF graphs, which are connected, consistent, and deadlock-free. This generation algorithm can be used to benchmark novel SADF analysis, transformation, and implementation algorithms. The user can restrict relevant properties of the generated graph (e.g., limit port rates, or construct only acyclic or strongly connected graphs). A set of command line tools as well as a $\mathrm{C} / \mathrm{C}++$ API implemented all algorithms used. The rich set of algorithms offered by SDF3, makes it a versatile tool set for the development of novel dataflow-based design approaches.

The predictable design flow contains four phases. Phase 1 aims to determine the memory that will be allocated for each actor and channel of the application graph on the tiles of the target platform. Phase 2 computes the minimal bandwidth needed by the application and the maximal latency on the NoC. Phase 3 binds the actors and channels of the application graph to the tiles of the NoC-MPSoC target platform. The arbitration is based on TDMA wheels for each processor. Phase 4 schedules the communication on the NoC, given the actors and channels mappings. When it is successful, the output of the predictable design flow is a NoC-MPSoC configuration that satisfies the throughput constraint. The configuration contains a mapping of the actors and channels of the SDF graph to the tiles and connections of the NoC-MPSoC destination platform. The mapping is modeled by applying transformations to the application graph, in order to model the binding of actors and channels to tiles and connections. The SDF3 tool has a conservative approach, which means that it will assume the 
Table 4. Test sequences used on experimentations.

\begin{tabular}{cccc}
\hline Class & Séquence & QP & Number of frames \\
\hline A & Traffic & 22 & 150 \\
B & Cactus & 22 & 500 \\
C & Party Scene & 22 & 500 \\
D & BQ Square & 22 & 600 \\
E & Kristen And Sara & 22 & 600 \\
F & China Speed & 22 & 500 \\
\hline
\end{tabular}

worst-case scenario at any stage of the analysis. The constraint that the SDF graph must respect is the bit rate that must not be below 25 frames per second. Therefore, 25 iterations per second because the decoder decompresses an image by iteration. The value of the constraint will therefore be 0.025 iterations pertime unit (ms).

\subsection{Actor's Execution Times and Space Memory}

Execution times of actors are the first information needed for the modeling. It is expressed in abstract time units used by the sdf 3 flow command. The designer is thus free to map this abstract unit to a "manly" unit. The time unit used in this work is the processor cycle. To get actors' execution times, several techniques are used. Some techniques use WCET tools or binaries; other uses simulation and other uses measurement and experimentations. In this work, we have done several profiling experimentations on the target platform (Zedboard) to calculate the WCET of each actor. Table 5 presents sequences execution time and throughput for all classes on the Zed board platform. Figure 7 presents the individual execution times for actors of HEVC Decoder (HM test model application).

In the rest of this paper, we present the experimental values applied to bits of class $\mathrm{C}(832 \times 480$ pixels $)$.

We will work with the largest execution time for the AI configuration (worst case), which is equal to 307.414 seconds, and after division on 500 we will have 0.615 seconds (615 ms). In Tables 6-8, we present actors execution time, size of memory and size of tokens exchanged between actors for AI configuration.

For the RA, LD, LP configurations, the largest execution time of the three configurations is 176.658 seconds and after division on 500 will have 0.3533 seconds (354 ms), Actors Execution time (ms)-RA/LP/LB configuration

The memory space sizes for RC, MC, IP, SAO and DB are 400,000 bytes because they manipulate variables that contain whole frames while the remainders are 5000 bytes because they only manipulate CTUs and then their variables are smaller.

To model the HEVC decoder for all configurations in class $\mathrm{C}$ (our uses case in experimentations), we use FSM-based-SADF extension. It is the most suitable tool to describe the application. 
Table 5. Sequences execution time and throughput for all classes on the Zedboard platform.

\begin{tabular}{|c|c|c|c|}
\hline Class & Configuration & Execution Time (s) & Throughput (f/s) \\
\hline \multirow{4}{*}{ A } & AI & 610.68 & 0.25 \\
\hline & RA & 293.25 & 0.51 \\
\hline & LP & 310.86 & 0.48 \\
\hline & LB & 333.82 & 0.45 \\
\hline \multirow{4}{*}{ B } & AI & 1074.852 & 0.47 \\
\hline & RA & 532.566 & 0.94 \\
\hline & LP & 595.297 & 0.84 \\
\hline & LB & 609.094 & 0.82 \\
\hline \multirow{4}{*}{$\mathrm{C}$} & AI & 307.414 & 1.63 \\
\hline & RA & 153.405 & 3.26 \\
\hline & LP & 176.658 & 2.83 \\
\hline & LB & 174.779 & 2.86 \\
\hline \multirow{4}{*}{$\mathrm{D}$} & AI & 86.741 & 6.92 \\
\hline & RA & 48.555 & 12.36 \\
\hline & LP & 55.283 & 10.85 \\
\hline & LB & 55.67 & 10.78 \\
\hline \multirow{4}{*}{$\mathrm{E}$} & AI & 393.206 & 1.53 \\
\hline & RA & 171.72 & 3.49 \\
\hline & LP & 175.796 & 3.41 \\
\hline & $\mathrm{LB}$ & 190.186 & 3.15 \\
\hline \multirow{4}{*}{ F } & AI & 382.396 & 1.31 \\
\hline & RA & 216.837 & 2.31 \\
\hline & LP & 217.949 & 2.29 \\
\hline & LB & 226.296 & 2.21 \\
\hline
\end{tabular}

The SDF3 tool requires an $\mathrm{xml}$ file that describes all information collected from profiling step (such memory sizes, actors execution times, scenarios, transitions between scenarios...). It is essential to adapt this information to the syntax imposed by the sdf3 because there are many rules to follow:

- Sizes must be in bytes.

- A same unit of time must be used along the description.

- The execution times introduced are just for a single iteration.

The extension of SDF, FSM-based-SADF, which we exploited in our work, requires that the values introduced in the XML description of the application are values that describe the worst case. In this case, the measurement and estimation results in terms of bit rate (throughput) and memory space provided by SDF3 will never be exceeded even if there are other applications that run simultaneously on the same platform.

For the class C we have 8 scenarios: I, BP0, BP400, BP600, BP800, BP1000, BP1300, BP1560. After that, we can notice the existence of the values of the rates, which are denoted by integers (when the value is constant for all the scenarios) or by letters (a, b, c, d when the value is a variable according to the scenarios), 
Actors Execution Time (\%) - Al configuration (Zedboard ARM Processors)

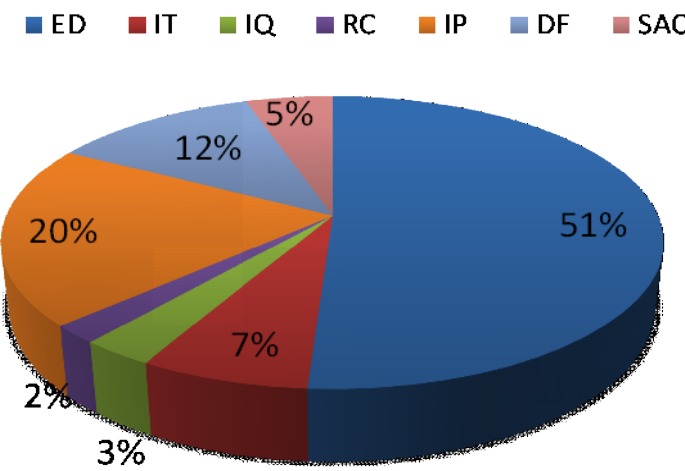

(a)
Actors Execution Time (\%) - RA configuration (Zedboard - ARM Processors )

$\square \mathrm{ED} \square \mathrm{IT} \square \mathrm{IQ} \square \mathrm{RC} \square \mathrm{MC} \square \mathrm{IP} \square \mathrm{DF} \square \mathrm{SAO}$

$4 \%$

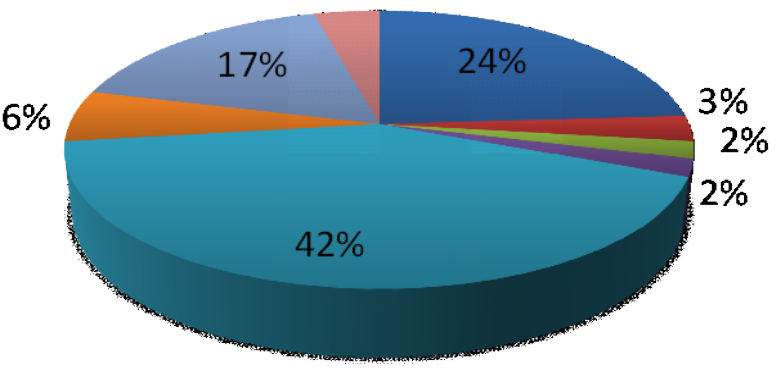

(b)

Figure 7. Percentages of execution time for each actor (for AI and RA configuration).

Table 6. Actors Execution time (ms)_AI configuration.

(a)

\begin{tabular}{cccccccc}
\hline ED & RC & IQ & IT & MC & IP & DF & SAO \\
\hline 313.56 & 12.30 & 18.44 & 43.04 & inactif & 122.97 & 73.78 & 30.74 \\
\hline
\end{tabular}

(b)

\begin{tabular}{cccccccc}
\hline ED & RC & IQ & IT & MC & IP & DF & SAO \\
\hline 84.80 & 7.07 & 7.07 & 10.60 & 148.39 & 21.20 & 60.06 & 14.13 \\
\hline
\end{tabular}

Table 7. Sizes of memory spaces consumed to store the internal state of each actor (byte).

\begin{tabular}{cccccccc}
\hline ED & RC & MC & IQ & IT & DB & IP & SAO \\
\hline 5000 & 400,000 & 400,000 & 5000 & 5000 & 400,000 & 400,000 & 400,000 \\
\hline
\end{tabular}

Table 8. Sizes of tokens (byte).

\begin{tabular}{cccccccccccc}
\hline DE2IQ & DE2MC & DE2IP & DE2SAO & IQ2IT & IT2RC & TA23DF & DF2SAO & MC2RC & SAO2MC & TA23IP & IP2RC \\
\hline 16,384 & 12 & 16,384 & 5 & 16,384 & 16,384 & $1,597,440$ & $1,597,440$ & $1,597,440$ & $1,597,440$ & $1,597,440$ & $1,597,440$ \\
\hline
\end{tabular}

Table 9 presents the values of rates according to the execution scenario. $\mathrm{Y}$ is the number of CTUs that are the references in an I frame. i.e. CTUs that will be used to generate the others (they will be passed in the residue). $\mathrm{X}$ is the number of blocks that have motion vectors. So $(1560-\mathrm{x})$ is the number of CTUs that will be passed in the residue (case of frames of types $\mathrm{P}$ and $\mathrm{B}$ ).

Table 10 illustrates the throughput variation of the decoding application according to the available memory size.

When we increase the memory space dedicated to input output channels for different actors, the throughput increases.

\section{Conclusions}

In this paper, we presented an approach oriented performance estimation for MPSoC Co-design flow. This approach is structured around four models: 
Table 9. Scenarios.

\begin{tabular}{ccc}
\hline & \multicolumn{2}{c}{ Scenarios } \\
\cline { 2 - 3 } & Iy & BPx \\
\hline a & Y & $1560-\mathrm{x}$ \\
b & 0 & $\mathrm{X}$ \\
c & 1 & 0 \\
d & 1 & 0 \\
\hline
\end{tabular}

Table 10. Throughputs variation.

\begin{tabular}{|c|c|}
\hline Total memory size (MB) & Throughput (frame/s) \\
\hline 0.85379 & 5.1 \\
\hline 21.099652 & 5.1 \\
\hline 21.09975 & 5.1 \\
\hline 21.427566 & 5.1 \\
\hline 21.427622 & 5.1 \\
\hline 21.755438 & 5.1 \\
\hline 312.548904 & 64.5 \\
\hline 312.630914 & 64.5 \\
\hline 312.794898 & 64.5 \\
\hline 312.958882 & 64.5 \\
\hline 313.122866 & 64.5 \\
\hline 313.28685 & 64.5 \\
\hline 521.159654 & 106.1 \\
\hline 521.323638 & 106.1 \\
\hline 521.487622 & 106.1 \\
\hline 521.651606 & 106.1 \\
\hline 521.81559 & 106.1 \\
\hline 521.979574 & 106.1 \\
\hline 729.852378 & 147.7 \\
\hline 730.016362 & 147.7 \\
\hline 730.180346 & 147.7 \\
\hline 730.34433 & 147.7 \\
\hline 730.508314 & 147.7 \\
\hline 730.672298 & 147.7 \\
\hline 938.545102 & 189.3 \\
\hline 938.709086 & 189.3 \\
\hline 938.87307 & 189.3 \\
\hline 939.037054 & 189.3 \\
\hline 939.201038 & 189.3 \\
\hline 939.365022 & 189.3 \\
\hline
\end{tabular}


Functional Model MF (describing the application behavior), Architecture Model AM (describing the platform targets in number of processors, cache memory, communication system Bus/NoC), Constraint Model CM and Performance Model PM (execution time representation). The two new models introduced are the PM and the CM. The Constraint Model (CM) describes the functional constraints of the application and non-functional constraints of the target platform. The Performance Model PM describes a performance evaluation of system in every abstraction level. An extension of Khan's model is used. It is based on addition of relative annotations to execution times and to the size of the data exchanged of the parallel model. Experimentation is achieved, in this context, on the HEVC/h.265 decoder and the Zedboard platform of Xilinx.

The HEVC/h.265 video decoder is modeled with SADF based FSM in order to solve problems of placing and scheduling this application on an embedded architecture. This is done by identifying actor dependencies, number of data exchanged (tokens), execution time and space memory of the decoder applied on C class of bitstreams.

A high-level performance analysis is performed to find an optimal balance between the decoding efficiency and the implementation cost allowing for a complexity reduction at a system level. For an optimal use of the HEVC tools, the best configuration parameters are obtained. For this cost-efficient configuration, the absolute complexity values, the memory and task level profiling results confirmed the big challenge needed for its effective implementation. For such implementation, a multiprocessor approach is needed to share the decoding application execution time between several processors for achieving better execution performances and real time decoding.

\section{References}

[1] Jantsch, A. and Sander, I. (2005) Models of Computation and Languages for Embedded System Design. Computers and Digital Techniques, 152, 114-129.

[2] Lee, E.A. and Neuendorffer, S. (2005) Concurrent Models of Computation for Embedded Software. Computers and Digital Techniques, 152, 239-250.

[3] Hopcroft, J. and Ullman, J. (1979) Introduction to Automata Theory, Languages, and Computation. Addison-Wesley Publishing Company, Reading.

[4] Lee, E. and Messerschmitt, D. (1987) Synchronous Data Flow. IEEE Proceedings, 75, 1235-1245.

[5] Sriram, S. and Bhattacharyya, S.S. (2000) Embedded Multiprocessors: Scheduling and Synchronization. Marcel Dekker, Inc., New York.

[6] Bhattacharyya, S.S., Deprettere, F., Leupers, R. and Takala, J. (2013) Handbool of Signal Processing System. London.

[7] Stuijk, S. (2007) Predictable Mapping of Streaming Applications on Multiprocessors. PhD Thesis, Eindhoven University of Technology.

[8] Moonen, A., Bekooij, M. and van Meerbergen, J. (2004) Timing Analysis Model for Network Based Multiprocessor Systems. Proceedings of Progress Symposium on Embedded Systems, 122-130. 
[9] Bennour, I., Sebai, D. and Jemai, A. (2010) Modeling SW to HW Task Migration for MPSOC Performance Analysis. DTIS.

[10] Mesman, K.B., Theelen, B., Corporaal, H. and Ha, Y. (2008) Analyzing Composability of Applications on MPSoC Platforms. Journal of Systems Architecture, 54, 369-383.

[11] Wiggers, M.H., Kavaldjiev, N., Smit, G.J.M. and Jansen, P.G. (2005) Architecture Design Space Exploration for Streaming Applications through Timing Analysis. Centre for Telematics and Information Technology, University of Twente, Enschede, Technical Report TR-CTIT-05-36.

[12] Shabbir, A., Kumar, A., Stuijk, S., Mesmana, B. and Corporaal, H. (2010) CAMPSoC: An Automated Design Flow for Predictable Multi-Processor Architectures for Multiple Applications. Journal of Systems Architecture-Embedded Systems Design, 56, 265-277.

[13] Geilen, M. (2010) Synchronous Dataflow Scenarios. ACM Transactions on Embedded Computing Systems, 10, 16:1-16:31.

[14] Stuijk, S., et al. (2011) Scenario-Aware Dataflow: Modeling, Analysis and Implementation of Dynamic Applications. 11 th International Conference.

[15] Phan, L.T.X., Chakraborty, S. and Thiagarajan, P.S. (2008) A Multi-Mode Real-Time Calculus. Proceedings of the 2008 Real-Time Systems Symposium, Washington DC, 59-69.

[16] Thiele, L. and Stoimenov, N. (2009) Modular Performance Analysis of Cyclic Dataow Graphs. Proceedings of the 7 th ACM International Conference on Embedded Software, New York, 127-136.

[17] Theelen, B.D., Geilen, M., Basten, T., Voeten, J., Gheorghita, S.V. and Stuijk, S. (2006) A Scenario-Aware Data Model for Combined Long-Run Average and Worst-Case Performance Analysis. Memocode, 185-194.

[18] Poplavko, P., Basten, T. and van Meerbergen, J. (2007) Execution-Time Prediction for Dynamic Streaming Applications with Task-Level Parallelism. Proceedings of the 10 th Euromicro Conference on Digital System Design Architectures, Methods and Tools, Washington DC, 228-235.

[19] Geilen, M. (2009) Synchronous Dataflow Scenarios. Transactions on Embedded Computing Systems, Special Issue on Model-Driven Embedded-System Design.

[20] Ehrlich, P. and Radke, S. (2013) Energy-Aware Software Development for Embedded Systems in HW/SW Co-Design. 16th International Symposium on Design and Diagnostics of Electronic Circuits \& Systems.

[21] Kai, H., Xio-xu, Z., Si-wen, X., et al. (2015) Profiling and Annotation Combined Method for Multimedia Application Specific MPSoC Performance Estimation. Springer-Verlag, Berlin, Heidelberg.

[22] Smei, H., Smiri, K. and Jemai, A. (2017) Profiling of HEVC Decoder Application in a Co-Design flow. 6th International Colloquium in Applied Research and Technology Transfer.

[23] Kahn, G. (1974) The Semantics of a Simple Language for Parallel Programming. Information Processing 74: Proceedings of the IFIP Congress 74, Stockholm, August 1974, 471-475.

[24] Bebelis, V. (n.d.) Boolean Parametric Data Flow. Streaming Day, V. BEBELIS (INRIA) BPDF. http://streaming.conf.citi-lab.fr/streaming_bebelis.pdf

[25] Miyazaki, T. (1997) Code Generation by using Integer-Controlled Dataflow Graph. Acoustics, Speech, and Signal Processing, ICASSP-97. 
[26] Wauters, P., Engels, M., Lauwereins, R. and Peperstraete, J.A. (1996) Cyclo-Dynamic Dataflow. Parallel and Distributed Processing.

[27] Lee, E.A. and Messerschmitt, D.G. (1987) Synchronous Data Flow. Proceedings of IEEE, 75, 1235-1245.

[28] Lee, E.A. and Messerschmitt, D.G. (1987) Static Scheduling of Synchronous Data Flow Programs for Digital Signal Processing. IEEE Transactions on Computers, 36, 24-35.

[29] Design and Implementation of Next Generation Video Coding Systems (H.265/HEVC Tutorial), Vivienne Sze, ISCAS Tutorial, 2014.

[30] Smei, H. and Jemai, A. (2016) Pipelining the HEVC Decoder on ZedBoard Platform. International Design \& Test Symposium IDT.

[31] Wiegand, T., et al. (2003) Overview of the H.264/AVC Video Coding Standard. IEEE Transactions on Circuits and Systems for Video Technology, 13, 560-576.

[32] Valgrind Web. http://valgrind.org/

[33] https://sourceware.org/binutils/docs-2.16/gprof/

[34] https://wiki.gnome.org/Apps/MemProf

[35] Bossen, F. (2012) Common Test Conditions and Software Reference Configurations. 9 th Meeting of the JCT-VC in Geneva.

[36] BSD Licence HEVC Decoder (HM). https://hevc.hhi.fraunhofer.de https://github.com/bbc/vc2-reference

[37] Theelen, B.D., Geilen, M.C.W., Stuijk, S., Gheorghita, S.V., Basten, T., Voeten, J.P.M. and Ghamarian, A.H. (2008) Scenario-Aware Dataflow, ES Reports.

[38] Zedboard Plateform. http://www.zedboard.org

[39] Xilinx, Inc. Zynq-7000 All Programmable SoC Technical Reference Manual. http://www.xilinx.com/support/documentation/user_guides/ug585-Zynq-7000-TR M.pdf

[40] Stuijk, S., Geilen, M. and Basten, T. (2006) SDF3: SDF for Free. Application of Concurrency to System Design, 276-278. 